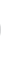

\section{A wider pathological network underlying breathlessness and respiratory failure in amyotrophic lateral sclerosis}

\author{
Kyle T.S. Pattinson and Martin R. Turner
}

Affiliation: Nuffield Dept of Clinical Neurosciences, University of Oxford, John Radcliffe Hospital, Oxford, UK.

Correspondence: Kyle T.S. Pattinson, Nuffield Dept of Clinical Neurosciences, University of Oxford, John Radcliffe Hospital, Oxford, OX3 9DU, UK. E-mail: kyle.pattinsonanda.ox.ac.uk

\footnotetext{
0
} @ERSpublications

Neurophysiology studies in ALS may help us better understand breathlessness in primary respiratory disease http://ow.ly/ZkQLV

The adult-onset neurodegenerative disorder amyotrophic lateral sclerosis (ALS) is characterised by progressive dysfunction of upper motor neurons of the corticospinal tract, brainstem nuclear and lower motor neurons of the spinal cord anterior horns. There is clinicopathological and genetic overlap with frontotemporal dementia, and an emerging view of ALS as a syndrome [1]. The majority of patients present with either focal limb or bulbar muscle weakness, which then spreads at a variable rate to other body regions [2]. Median survival in ALS is 30 months from symptom onset, with most deaths associated with type II respiratory failure [3]. In the majority of ALS patients, respiratory failure emerges insidiously after a variable period of progressive limb or bulbar muscle weakness. Symptoms are highly variable, but include breathlessness, often with orthopnoea, sleep fragmentation (with nocturnal desaturations detectable on oximetry), daytime somnolence and other systemic symptoms associated with chronic hypercapnia.

The novel study by Georges et al. [4], in this issue of the European Respiratory Journal, studied higher cerebral activity in ALS patients with respiratory failure. The authors raise the possibility that cortically driven responses to diaphragm weakness, which are also associated with accessory muscle activation, might have the undesirable effect of increasing breathlessness. In a technically challenging study, a small group of ALS patients with respiratory failure underwent electroencephalography to detect pre-inspiratory potentials (PIPs), along with electromyography of inspiratory neck muscles, during both spontaneous breathing and when using noninvasive ventilation (NIV). The authors noted that NIV appeared to attenuate the PIPs found in more than half of patients during spontaneous breathing. These PIPs were identified in the $\mathrm{Cz}$ position, most probably reflecting brain activity in sensory and motor areas. NIV reduced the inspiratory neck muscle activity seen in all patients, and importantly, also their breathlessness.

NIV prolongs survival as well as improving the symptoms of respiratory insufficiency in ALS, including breathlessness [5]. Diaphragm weakness is assumed to be a principal driver of respiratory insufficiency in ALS, with orthopnoea very common in the advanced stages. An accurate measure of diaphragm strength using oesophageal pressure has been correlated with survival in ALS [6]. Other studies have shown that the use of accessory muscles is a sensitive and specific surrogate marker for diaphragm weakness [7], with a specific link to the electromyographic detection of denervation in paraspinal muscles [8]. Vital capacity is the commonly used surrogate to identify ALS patients developing respiratory failure in the clinic,

Received: Feb 122016 | Accepted: March 042016

Support statement: This work was supported by the Medical Research Council (grant number: MR/K01014X/1) and the National Institute of Health Research, Oxford Biomedical Research Centre. Funding information for this article has been deposited with FundRef.

Conflict of interest: None declared.

Copyright OERS 2016 
although sniff nasal pressure has been shown to be more sensitive and is particularly useful for those with bulbar dysfunction [9].

ALS is associated with widespread cortical and brainstem pathology, but less is known about the relative contribution of the wider respiratory control network in ALS, with the suggestion that there may be compensatory neuronal plasticity [10]. Sleep disordered breathing is common in ALS and appears driven by central apnoeic events [11]. Lesions of the central pattern-generating neurons of the brainstem pre-Bötzinger complex produce similar deficits [12], although this region has not yet been the subject of dedicated study in ALS.

The authors are clear about the limitations of their study, principally the use of a small patient group with symptomatically advanced disease. The six patients in this study who did not show PIPs were generally more impaired, with longer disease duration and more severe respiratory impairment, but reported less breathlessness. The authors can only speculate whether this sub-group has decompensated, suffered an effect of more chronic NIV use or has an unrelated basis for what is increasingly recognised to be a clinically heterogeneous syndrome.

Cortical activity in relation to sleep disordered breathing in ALS is an obvious area in which this study offers new avenues for exploration, and potentially modulation. It has been suggested that the only licensed, modestly disease-modifying drug for ALS, namely riluzole, might have a specific beneficial role in modulating sleep disordered breathing based on effects on neuronal rhythmicity in rodent models [13].

The authors identify the need to explore the relationship of their findings with the expanding awareness of frontotemporal cognitive and behavioural cortical deficits inherent to this multisystem neurodegenerative disorder. Breathlessness is likely to worsen already impaired cognitive performance via reallocation of limited attentional resources [14]. Beyond ALS, this may have relevance to primary respiratory disorders. In chronic obstructive pulmonary disease (COPD) there is some evidence that pulmonary rehabilitation improves cognitive performance [15], which may be partly due to improvements in breathlessness. Therefore the link between breathlessness and cognitive dysfunction is particularly worthy of further investigation.

More broadly, there is increasing realisation that the sensation and impact of breathlessness is considerably more complex than simply increased neural traffic between the lungs and the sensory cortex [16-18]. Abnormal respiratory signalling is shaped by a variety of emotional and psychological processes in different parts of the brain, the interactions of which lead to the sensations of breathlessness. Drawing a parallel from $\mathrm{COPD}$, there is mounting evidence that many of the beneficial effects of pulmonary rehabilitation derive from relieving anxiety about breathlessness rather than the physical sensations of breathlessness [19, 20]. Thus in the context of ALS a potential beneficial strategy may be to consider anxiety about breathlessness independently, especially once medical therapy is optimised [21]. Observations on breathlessness anxiety in COPD suggest that fear conditioning related to learned negative associations between everyday situations and dyspnoea exacerbate the downward spiral of breathlessness and inactivity. Neuroimaging studies testing this are starting to emerge [22, 23], and implicate emotional processes in the medial prefrontal cortex contributing to chronic breathlessness. Whether neurodegeneration in frontal and other emotion-processing brain regions specifically contributes to breathlessness in ALS remains unknown. In the present dataset, it is clear than NIV successfully attenuated the affective dimensions of breathlessness, presumably reducing the work of breathing to such an extent that the PIPs disappeared.

Measurements of the affective components of breathlessness combined with neurophysiology and neuroimaging have great potential to help individualise the treatment of breathlessness and have potential as a powerful stratification tool, whether it be in ALS, COPD, cancer or asthma. Notably, this study quantitatively examined the subjective impact of breathlessness. The authors used the recently developed Multidimensional Dyspnea Profile (MDP) [24], revealing measurable anxiety about breathlessness in both groups during spontaneous breathing, which was relieved by NIV. These findings echo those of a qualitative study [25].

Although the MDP has been designed primarily as a research tool [24], an alternative approach for clinical (and research) use is the Dyspnoea-12 (D-12) questionnaire [26]. The D-12 can be split into affective and physical components. The D-12 has been validated in clinical practice with a minimally clinically important difference of three units for the overall score [27]. A detailed comparison of the two instruments is discussed by BANZETT et al. [24]. To fully evaluate how measuring the affective component of breathlessness will benefit patients, wider use of instruments such as these is now necessary in clinical practice, in conjunction with clinical research and quality improvement projects.

Future research using electroencephalography or functional neuroimaging [28] might specifically address the mechanisms by which emotions influence breathlessness processing in ALS, with the potential for 
novel therapeutic or palliative strategies. Thus the study of Georges et al. [4] broadens our insight into the neural networks underpinning the respiratory control system, and may lead to a deeper understanding of the neurophysiology of breathlessness across a more diverse range of pathology.

\section{References}

1 Turner MR, Swash M. The expanding syndrome of amyotrophic lateral sclerosis: a clinical and molecular odyssey. J Neurol Neurosurg Psychiatry 2015; 86: 667-673.

2 Kiernan MC, Vucic S, Cheah BC, et al. Amyotrophic lateral sclerosis. Lancet 2011; 377: 942-955.

3 Kurian KM, Forbes RB, Colville S, et al. Cause of death and clinical grading criteria in a cohort of amyotrophic lateral sclerosis cases undergoing autopsy from the Scottish Motor Neurone Disease Register. J Neurol Neurosurg Psychiatry 2009; 80: 84-87.

4 Georges M, Moraviec E, Raux M, et al. Cortical drive to breathe in amyotrophic lateral sclerosis: a dyspnoea-worsening defence? Eur Respir J 2016; 47: 1818-1828.

5 Bourke SC, Tomlinson M, Williams TL, et al. Effects of non-invasive ventilation on survival and quality of life in patients with amyotrophic lateral sclerosis: a randomised controlled trial. Lancet Neurol 2006; 5: 140-147.

6 Vitacca M, Clini E, Facchetti D, et al. Breathing pattern and respiratory mechanics in patients with amyotrophic lateral sclerosis. Eur Respir J 1997; 10: 1614-1621.

7 Lechtzin N, Wiener CM, Shade DM, et al. Spirometry in the supine position improves the detection of diaphragmatic weakness in patients with amyotrophic lateral sclerosis. Chest 2002; 121: 436-442.

8 de Carvalho M, Pinto S, Swash M. Association of paraspinal and diaphragm denervation in ALS. Amyotroph Lateral Scler 2010; 11: 63-66.

9 Lyall RA, Donaldson N, Polkey MI, et al. Respiratory muscle strength and ventilatory failure in amyotrophic lateral sclerosis. Brain 2001; 124: 2000-2013.

10 Nichols NL, Van Dyke J, Nashold L, et al. Ventilatory control in ALS. Respir Physiol Neurobiol 2013; 189: 429-437.

11 Bourke SC, Shaw PJ, Gibson GJ. Respiratory function vs sleep-disordered breathing as predictors of QOL in ALS. Neurology 2001; 57: 2040-2044.

12 McKay LC, Janczewski WA, Feldman JL. Sleep-disordered breathing after targeted ablation of preBötzinger complex neurons. Nat Neurosci 2005; 8: 1142-1144.

13 Bellingham MC. A review of the neural mechanisms of action and clinical efficiency of riluzole in treating amyotrophic lateral sclerosis: what have we learned in the last decade? CNS Neurosci Ther 2011; 17: 4-31.

14 Eccleston C, Crombez G. Pain demands attention: a cognitive-affective model of the interruptive function of pain. Psychol Bull 1999; 125: 356-366.

15 Dodd JW, Getov SV, Jones PW. Cognitive function in COPD. Eur Respir J 2010; 35: 913-922.

16 Hayen A, Herigstad M, Pattinson KT. Understanding dyspnea as a complex individual experience. Maturitas 2013; 76: $45-50$.

17 Herigstad M, Hayen A, Wiech K, et al. Dyspnoea and the brain. Respir Med 2011; 105: 809-817.

18 Pattinson KT, Johnson MJ. Neuroimaging of central breathlessness mechanisms. Curr Opin Support Palliat Care 2014; 8: 225-233.

19 Carrieri-Kohlman V, Gormley JM, Eiser S, et al. Dyspnea and the affective response during exercise training in obstructive pulmonary disease. Nurs Res 2001; 50: 136-146.

20 Herigstad M, Hayen A, Reinecke A, et al. Development of a dyspnoea word cue set for studies of emotional processing in COPD. Respir Physiol Neurobiol 2016; 223: 37-42.

21 Currow DC, Abernethy AP, Allcroft P, et al. The need to research refractory breathlessness. Eur Respir J 2016; 47: 342-343.

22 Herigstad M, Hayen A, Evans E, et al. Dyspnea-related cues engage the prefrontal cortex: evidence from functional brain imaging in COPD. Chest 2015; 148: 953-961.

23 Faull OK, Jenkinson M, Ezra M, et al. Conditioned respiratory threat in the subdivisions of the human periaqueductal gray. Elife 2016; 10.7554/eLife.12047.

24 Banzett RB, O’Donnell CR, Guilfoyle TE, et al. Multidimensional Dyspnea Profile: an instrument for clinical and laboratory research. Eur Respir J 2015; 45: 1681-1691.

25 Gysels MH, Higginson IJ. The lived experience of breathlessness and its implications for care: a qualitative comparison in cancer, COPD, heart failure and MND. BMC Palliat Care 2011; 10: 15.

26 Yorke J, Moosavi SH, Shuldham C, et al. Quantification of dyspnoea using descriptors: development and initial testing of the Dyspnoea-12. Thorax 2010; 65: 21-26.

27 Yorke J, Lloyd-Williams M, Smith J, et al. Management of the respiratory distress symptom cluster in lung cancer: a randomised controlled feasibility trial. Support Care Cancer 2015; 23: 3373-3384.

28 Pattinson K. Functional brain imaging in respiratory medicine. Thorax 2015; 70: 598-600. 